\title{
Validation of Performance Homogeneity of Chan-Vese Model on Selected Tumour Cells
}

\author{
Justice Kwame Appati, University of Ghana, Ghana \\ iD https://orcid.org/0000-0003-2798-4524 \\ Franklin Iron Badzi, University of Ghana, Ghana \\ (iD) https://orcid.org/0000-0003-4975-6469 \\ Michael Agbo Tettey Soli, University of Ghana, Ghana \\ (iD) https://orcid.org/0000-0002-9985-000X \\ Stephane Jnr Nwolley, Npontu Technology, Ghana \\ (iD) https://orcid.org/0000-0003-1205-0526 \\ Ismail Wafaa Denwar, University of Ghana, Ghana \\ (iD https://orcid.org/0000-0002-5777-6163
}

\begin{abstract}
This study aims to analyze the Chan-Vese model's performance using a variety of tumor images. The processes involve the tumors' segmentation, detecting the tumors, identifying the segmented tumor region, and extracting the features before classification occurs. In the findings, the Chan-Vese model performed well with brain and breast tumor segmentation. The model on the skin performed poorly. The brain recorded DSC 0.6949903, Jaccard 0.532558; the time elapsed 7.389940 with an iteration of 100. The breast recorded a DSC of 0.554107, Jaccard 0.383228; the time elapsed 9.577161 with an iteration of 100. According to this study, a higher DSC does not signify a well-segmented image, as the breast had a lower DSC than the skin. The skin recorded a DSC of 0.620420, Jaccard 0.449717; the time elapsed 17.566681 with an iteration of 200.
\end{abstract}

\section{KEYWORDS}

Chan-Vese, Deformable Models, Dice Similarity Coefficient, Level Set, Tumor

\section{INTRODUCTION}

According to Rajendran \& Dhanasekaran (2012), deformable models are among the most widely employed techniques for segmentation especially in the field of medical image analysis. This technique is mainly used to identify tumor boundaries in Magnetic Resonance Imaging (MRI) images. Though various medical image modalities currently exist with great improvement in the analysis and diagnosis of patient's condition, MRI techniques are usually preferred as they are sufficient enough in capturing a number of soft tissues in the human body (Kumar \& Mankame, 2020). In instances where these images contain tumor, it volume becomes a good indicator to track and prepare suitable treatments for recovery. The quantification of this volume require segmentation which in practice is done manually 
by field expects. Although the manual segmentation can be accurate, it is time consuming and also require qualified professionals which in effect significantly limit the number of segmentation that can be accomplished in practice (Vorontsov, Tang, Roy, Pal, \& Kadoury, 2017). In the segmentation of the tumor, it can be classified as malignant or benign. The benign tumors are homogeneous in structure with the absence of cancerous cells, whereas the malignant tumors are cancerous cells (Sharif et al., 2020). These cancerous cells can rapture and rapidly spread to other parts of the human body making them life-threatening and the need to remove them as soon as detected (Sehgal, Goel, Mangipudi, Mehra, \& Tyagi, 2016). To detect these cells, methods like active contours become useful. Active contour models are subsets of deformable models which have been experimented broadly in image processing and are recognized as one of the most widely used and successful approaches for image segmentation. The class of region-based active contour models works primarily by incorporating special functions called the level set. These functions are deformed under well defined energy functions which traces the evolving curve towards a targeted boundary. Although these integrated functions are incredibly robust for sophisticated medical images, they suffer in accuracy against the contour curve's initialization.. The Chan-Vese model as a variant of the region-based active contour models has gained considerable interest in various image segmentation techniques (Zawish, Siyal, Ahmed, Khalil, \& Memon, 2019) despite the generally known problem. Extensive investigations has been made in recent years with their integration to Computer-Aided Detection (CAD) systems for tumor segmentation making it an interesting tool to further investigate their performance across selected tumour cells in this study. Since the manual delineation of tumors is an arduous process with variations in results; it is therefore critical to develop an automated model for segmentation (Kharote, Sankhe, \& Patkar, 2019; Chahal, Pandey, \& Goel, 2020)).

In this study we seek to analyze the perfornance of the famous Chan-Vese model in an attempt to answer the question "Is Chan-Vese Model generally scalable across different tumor types?" To address this question, appropriate research data is required and study has shown that the predominate datasets sufficient for the current study are MIAS, Navoneel, Diaretdb0, and ISIC. In evaluating the performance of the model, the following metrics are used: Dice Similarity Coefficient (DSC) and Jaccard Index (JI).

\section{RELATED WORKS}

This section gives in chronological form activities in this field of study for the past few years to establish gaps and possibly improve term. In the study of Gosse, Jehan-Besson, Lecellier, \& Ruan (2017), authors quest to appraise the effectiveness of 2D and 3D region-based models. The dataset used is positron emission tomography (PET) images by the MICCAI PETSEG Challenge and the methods employed are region-based deformable models such as: Chan-Vese, Locally Adaptive Random Walk (LARW), Poisson 3D, and Random Walker. The metric for the evaluation of these models was the Dice Coefficients. In their findings, the Chan-Vese 3D model recorded a Dice Coefficients of 0.8158 , Poisson 3D recorded 0.7251, Random Walker (RW) recoded 0.7766 , and finally, LARW recorded 0.8014 . This illustrate a significant improvement of the segmentation from $2 \mathrm{D}$ to $3 \mathrm{D}$ and a validate Poisson law's implication in PET images. Nevertheless, the Chan-Vese model is the highest performing technique and delivers comparable outputs to the LARW technique. In the same year, Sultana, Blatt, Gilles, Rashid, \& Audette (2017) attempts to recognize the boundary of cranial nerves and the medial axis by segmenting the ten pairs of brainstem cranial nerves from CNIII (the oculomotor nerve) to CNXII (the Hypoglossal nerve). The dataset for training the model was MRI data but the methods employed were a medial axis identification algorithm and a 3D deformable 1-simplex discrete contour model. The metrics for evaluating the models were the Dice's coefficient, average distance, Hausdorff Distance, and the Mean Square Distance (MSD). The surface segmentation technique indicated a positive outcome of $82 \%$ Dice Coefficient between semi-automatic and manual outputs in their findings. Shil et al. (2017) also designed an automated scheme to address the intricacies of 
detecting brain tumors. The dataset used was MRI images of Brats13. The methods used were Otsu binarization, and $\mathrm{K}$ means clustering for segmentation. For feature extraction, the Discrete Wavelet Transform (DWT) coupled with Principle Component Analysis (PCA) was used while Support Vector Machine (SVM) was used for classification. The metrics for evaluation were sensitivity, specificity, and accuracy. Their findings demonstrated that the proposed scheme achieved a classification accuracy of $99.33 \%$, sensitivity $99.17 \%$, and specificity $100 \%$. The study of Abbasi \& Tajeripour (2017) also seek to detect brain tumors accurately to aid patient's recovery. In the study, BRATS 2013 (glioma images) was used. The histogram orientation gradient and the local binary patterns were the methods employed. The metrics for evaluation were the Dice and Jaccard. In their findings, the proposed framework is more effective in detecting brain tumors than other methods. Finally, with the study of Fiçici, Eroğul, \& Telatar (2017) brain tumor segmentation were perform automatically, as the manual approach is time-wasting and less accurate. The dataset employed were FLAIR, T1 Post Gadolinium (T1C), and T1 Pre Gadolinium, and MRI. The thresholding, skull stripping, and fuzzy $\mathrm{C}$ mean clustering techniques were used and evaluated using sensitivity, specificity, precision, accuracy, overlap (Jaccard Coefficient), and dice similarity. In their findings, the proposed algorithm, together with symmetry analysis, performed well experimentally.

In the year 2018, Bal, Banerjee, Sharma, \& Maitra (2018) seeks to detect brain tumors as high varieties of tumor tissues impede a precise diagnosis. The dataset was MR images of the BRATS: Multimodal Brain Tumor Segmentation Challenge, Whole Brain Atlas (WBA), and eHealth laboratory. The method employed was fuzzy-possibilistic C-means (FPCM), and K-means. The metrics for evaluation were the Similarity index, Jaccard index, False-negative volume function (FNVF), False positive volume function (FPVF), Solidity, and Roundness. In their findings, the proposed model Patched based K-means and fuzzy possibilistic C-means recoded a high similarity index of 0.91 . The Cellular automata on the other hand recorded 0.72 while the SVM recorded 0.86 . The Concatenated RFs, which was trained using asymmetry and first-order statistical features recorded 0.87 , and the Generative model that performs joint segmentation registration recorded 0.88. Shivhare, Sharma \& Singh (2018) built an automated model for the diagnosis of brain tumors to resolve challenges associated with manual diagnosis. The dataset employed was the BRATS 2015. The methods employed were hole-filling operations and parameter-free clustering algorithm and morphological dilation. The metric for the evaluation was the Dice Similarity Coefficient (DSC). In their findings, the outcome of the segmentation yielded $75 \%$ of the DSC for a tumor region after comparing it with the ground truth. Finally, the study of Kozegar, Soryani, Behnam, Salamati, \& Tan (2018) seeks to solve issues of automatically segmenting the breast's masses due to the large variety in shape, size, and texture of 3-D objects. They aim to automate 3-D breast ultrasound-the dataset of 50 masses, consisting of 12 benign and 38 malignant lesions. Two kinds of ABUS systems datasets were used: the ACUSON S2000 automated breast volume system scan and the SomoVu automated 3-D breast ultrasound system. The method employed is a Gaussian mixture model which resulted in a mean Dice of $0.74 \pm 0.19$, which outmatched the adaptive region growing with a mean Dice of $0.65 \pm 0.2$ ( $\mathrm{p}$-value $<0.02$ ). Furthermore, the resulting mean Dice significantly ( $\mathrm{p}$-value $<0.001$ ) outperformed the distance regularized level set evolution method $(0.52 \pm 0.27)$.

Subsequent to 2018, Shivhare, Kumar, \& Singh (2019) attempts to automate brain tumors' detection with a hybrid approach as the prediction is challenging manually with the shape of the tumor being unstructured. The dataset used is BRATS2015 MRI images. The methods used were active contour model and convex hull. In their findings, the proposed method is validated in terms of the Sensitivity, Positive Predictive Value (PPV), and Dice Similarity Coefficient. An average DSC score of $92 \%$ for a complete brain tumor, $81 \%$ for brain tumor core, and $83 \%$ for an enhanced brain tumor was recorded which yielded more than the conventional approach adopted. The study of Kharote, Sankhe, \& Patkar (2019) also segmented the prostate from Multiparametric Magnetic Resonance Imaging as segmentation of the prostate is a complicated process due to the extensive variations of unclear prostate boundaries in prostate shape. The database used T2-weighted prostate 
MR images of 184 subjects. The methods employed were Hidden Features and Deformable Model, which involves stacked sparse AE. The metrics used were mean absolute surface distance (MASD) and the dice similarity coefficient. In their findings, the model's DSC is $87.4 \% \pm 4.6 \%$, and MASD of 1.80 $\mathrm{mm}$. The outcome indicates that the features learned are more capable of MR prostate segmentation. The study of Zawish, Siyal, Ahmed, Khalil, \& Memon (2019) also seek to detect brain tumors by segmentation which is considered a challenging task due to the overlapping of tissues. The dataset used was MRI images from Brain Atlas research. The method employed was the Chan-Vese active contour and evaluated using the computational time. In their findings, the proposed model indicated promising segmentation results. The proposed model (Chan-Vese without contours) recorded the lowest time with 3.7s for segmentation with respect to existing models like FCM, Region growing, K-mean, and Hist-Thr with running time 7.2s, 5.5s, 4.8s, 3.4s, and 3.7s respectively. Furthermore, the study of Mohamed Sathik \& Synthiya Judith Gnanaselvi (2019) attempts to automate the detection of brain tumors as the diagnosis can be complicated. The dataset employed were MRI scans while employing the Radial Basis Function and Random Forest as the computational methods. The evaluation metrics were accuracy, MSE (Mean Square Error), and PSNR (Peak Signal-to-Noise-Ratio). In their findings, the Radial Basis Function and Random Forest recorded an accuracy of 68.61 and 77.58 respectively for Glioma. The Radial Basis Function and Random Forest also recorded an accuracy of 67.08 and 81.41 respectively for Meningioma a 67.5 and 86.38 respectively for Normal. Finally, Agarwal \& Bhardwaj (2019) focused on detecting brain tumors, specifically Gliomas, as considerable structural and spatial variation between tumors poses an automation challenge. The dataset used was BRATS challenge datasets with extreme learning machine (ELM) as the method. The metrics used for the evaluation were DSC, PPV and sensitivity. In their findings, the ELM recorded a Dice of 0.98, and the Regression Extreme Learning Machine(RELM-L00) recorded 0.96 on a BRATS 2012 and 0.99 for both methods when evaluated on the BRATS 2013 dataset.

Finally in 2020, the study of Badshah, Rabbani, \& Atta (2020) worked to reduce brain and breast tumors' manual workload by quantifying the deformed tissues. The database used was the MRI/ mammogram images (T1-w, T2-w, and FLAIR sequences) and the method employed was the Laplacian of Gaussian. The metrics for the evaluation were specificity, dice coefficient, Jaccard similarity, Accuracy, and sensitivity. In their findings, the proposed model obtains average values of $99.96 \%$ for Jaccard similarity, $99.88 \%$ for dice coefficient, $99.91 \%$ for Accuracy, $99.91 \%$ for sensitivity, and 99.95\% for specificity, which outperformed the existing techniques. Again, the study of Shrivastava \& Bharti (2020) attempted to detect breast cancer early, as tumor segmentation and classification accuracy can make diagnosis efficient. The dataset used were; RIDER breast MR Images and Digital Database for Screening Mammography (DDSM), and Mammographic Image Analysis Society (MIAS). The method used was Seeded Region Growing (SRG) and the metric used for evaluation was sensitivity known as the True Positive Fraction (TPF), specificity known as the False Negative Fraction (FNF), Dice Similarity Coefficient (DSC), Relative Overlap (RO), Sum of True Volume Fraction (SVTF), Misclassification Rate (MCR), Accuracy, False Positive Fraction(FPF), True Negative Fraction (TNF), Precision (P), and F-measure. In their findings, classification accuracy is better than previous techniques, which is $91.4 \%$. The hybrid technique detects the tumor's size and shape from both mammograms and MR Images and efficiently distinguishes the tumor as malignant or benign. Finally, the study of Khalil, Darwish, Ibrahim, \& Hassan (2020) detects brain tumors from 3D Magnetic Resonance Imaging (3D-MRI) as the brain tumor's size, structure, and form can be challenging. The dataset employed were the (BRATS) 2017 dataset and the method employed was the two-step dragonfly algorithm (DA) clustering technique. The metrics employed were Accuracy, Recall, and Precision. In their findings, the hybrid approach on 3D-MRI images from the multimodal brain tumor segmentation challenge BRATS2017 dataset indicates that the proposed technique for brain tumor segmentation shows potential just like the existing methods. 


\section{MATERIALS AND METHODS}

This section investigates the current approach employed in deformable models for tumor detection and discusses the various techniques used in detail. It also explains the data used for the study as well.

\section{Datasets}

In this study, the MIAS, Navoneel, and ISIC datasets were used to validate the Chan-Vese model. These datasets were specifically choosen as they were publicly available and widely used in this study area noted from the review done. Broadly, tumors cells can be associated with the brain, eye, skin, liver, and breast making it quite vasetile to study. However, as a proof of concept of the scalability of the famous Chan-Vese model the breast from MIAS, brain from Navoneel and skin from ISIS was considered.

\section{Image Preprocessing}

To carry out with the segementation process, data preprocessing is required. At that level, the images are converted to grayscale while the identified noise is filtered out with the anisotropic filters using Eqn. 1.

$$
\frac{\partial x}{\partial t}=\operatorname{div}(c(m, n, t) \nabla I)=\nabla c \cdot \nabla x+c(m, n, t) \nabla x
$$

where $\nabla c$ denotes the image gradient and $c(m, n, t)$ denotes the coefficient.

\section{Deformable Models}

\section{Chan-Vese Technique}

According to Lakshmi Priya, Saraswathi, \& Punitha Lakshmi (2019), Chan-Vese (CV) model as a segmentation tool is one of the state-of-the-art segmentation approach derived from gradient-based and thresholding segmenting technique. The approach employs an energy reduction function that can be formulated using a level set. As part of its formulation, it makes use of the Shah algorithm which is derived from edges and colors for the segmentation phase. The algorithm holistically, consists of some stages such as: feature extraction at different parameters and the optimization of its values. In optimizing the values, the position of the features are detected more accurately. These properties make them more advantageous over gradient-based approach as they resolve their inherent challenges. Figure 1 gives a detailed workflow of the current Chan-Vese segmentation algorithm. At the heart of Chan-Vese algorithm is the "fitting energy" function with an associated attribute derived from features such the image length and the color intensity. In the study of Z. Wang, Wang, Yang, Pan, \& Han (2018), the CV approach is generally implemented to images that require the segmentation of its regions into two: background and target. For a given image $I(x, y)$ on the domain $\Omega$ the $\mathrm{CV}$ algorithm seeks to decrease the energy function as mathematically expressed in Eqn. 2.

$$
\begin{aligned}
& \mathrm{E}\left(c_{1}, c_{2}, C\right)=u \cdot \text { Length }(C)+\lambda_{1} \int_{\Omega_{1}}\left|I(x, y)-c_{1}\right|^{2} d x d y \\
& +\lambda_{2} \int_{\Omega_{2}}\left|I(x, y)-c_{2}\right|^{2} d x d y I(x, y)+\operatorname{Area}(\operatorname{inside}(c))
\end{aligned}
$$

where $C$ denotes the curve, coefficients $\mathrm{v}, \lambda_{1}$, and $\lambda_{2}$ are fixed parameters, and constants $c_{1}$ and $c_{2}$ represents the average intensities in and out of the curve respectively. The length of $C$ and 
the area in $C$ are employed to check the boundary smoothness. Using the level set to denote $C$ as the Lipschitz function's $\phi(\mathrm{x}, \mathrm{y})$ zero-level set, Eqn. 2 can be rewritten as Eqn. 3.

$$
\begin{aligned}
& \mathrm{E}\left(c_{1}, c_{2}, \phi\right)=\lambda_{1} \int_{\Omega}\left|I(x, y)-C_{1}\right|^{2} H(\phi(x, y)) d x d y+\lambda_{2} \int_{\Omega} \mid I(x, y) \\
& -\left.c_{2}\right|^{2}(1-H(\phi(x, y))) d x d y+\mu \int_{\Omega} \delta(\phi(x, y))|\nabla \phi(x, y)| d x d y+v \int_{\Omega} H(\phi(x, y)) d x d y
\end{aligned}
$$

where $\delta(\phi)$ and $H(\phi)$ are the Dirac and Heaviside functions, respectively. Mainly, the regularized variant is expressed as shown in Eqn 4

$$
H(\phi)=\frac{1}{2}\left[1+\frac{2}{\pi} \arctan \left(\frac{\phi}{\varepsilon}\right)\right], \delta(\phi)=\frac{1}{\pi} \cdot \frac{\varepsilon}{\varepsilon^{2}+\phi^{2}}
$$

Maintaining $c_{1}$ and $c_{2}$ fixed, $E\left(c_{1}, c_{2}, C\right)$ is minimized with regards to $\phi(\mathrm{x}, \mathrm{y})$, as we derive the Euler-Lagrange equation for $\phi(\mathrm{x}, \mathrm{y})$. Parameterizing the descent position arbitrarily by time $t$, the formulation of the corresponding variation level set can be obtained as Eqn 5.

$$
\begin{aligned}
& \frac{\partial \phi}{\partial t}=\delta(\phi)\left\{\mu \bullet d i v\left(\frac{\nabla \phi}{|\nabla \phi|}\right)-v-\lambda_{1}\left[I(x, y)-c_{1}\right]^{2}+\lambda_{2}\left[I(x, y)-c_{2}\right]^{2}\right\} \\
& c_{1}=\frac{\int \Omega I(x, y) H(\phi) d x d y}{\int \Omega H(\phi) d x d y} \\
& c_{2}=\frac{\int \Omega I(x, y)[1-H(\phi)] d x d y}{\int \Omega[1-H(\phi)] d x d y} \\
& \phi(x, y, 0)=\phi_{0}(x, y)
\end{aligned}
$$

In Eqn. 4, the CV uses the local gradient details to check the curve's deformation movement and the contour curve evolution. Two issues arise with this approach which require attention when extracting the target leaf from a given MR/CT image. The first is the initialization, and the second is the failure for the curve to evolve. Resolution of this problem requires the initialization to be done at the center of the image for the evolving curve to halt when its curvature is stable for a particular period.

\section{Level Sets}

Level-Set methods have been widely employed to capture interface development, especially when the interface experiences extreme topological changes. It adopts Hamilton Jacob and Partial Differential Equations method to fix the segmentation issue. In the formulation of the level set, a closed contour $\mathrm{C} \subset \Omega$ is usually denoted by zero level set implicitly as a Lipschitz function $\phi: \Omega \rightarrow \mathrm{R}$. The signed distance function (SDF, $\nabla=\phi 1$ ) denoting the surface is usually the appropriate choice of the active contour models and is expressed as Eqn. 6.

$$
\phi(x, t)=\left\{\begin{array}{cc}
\operatorname{dist}\left(x, C_{s}\right) & \text { if } \phi(x)>0 \\
0 & \text { if } \phi(x)=0 \\
-\operatorname{dist}\left(x, C_{r}\right) & \text { if } \phi(x)<0
\end{array}\right.
$$

where the evolving curve $C_{t}$ at time $t$ is the shortest; $\operatorname{dist}\left(\mathrm{x}, C_{t}\right)$ is Euclidean distance from the pixel position $x$. Generally, it enables the curve to go through topological changes by resolving the 
energy functional solution spontaneously. The level set function $\phi$ take negative and positive values inside and outside the contour $C$, and further deduce the model's energy function as shown in Eqn. 7.

$$
F\left(\phi, c_{p}, c_{2}\right)=\sum_{i=1}^{2}\left(\lambda_{j} \int_{\Omega_{2}}\left(\int K_{\sigma}(x-y)\left(f(y)-c_{i}\right)^{2} d y\right) M_{i}^{c}(\phi(x)) d x\right)+\mu \int_{\Omega}\left|\nabla H_{\epsilon}(\phi(x))\right| d x
$$

where $M_{i}^{c}(\bullet)$ is defined in Eqn. 8

$M_{1}^{c}(\phi(x))=H_{c}(\phi)=1 / 2 \times(1+2 / \pi \times \arctan (\phi(x) / \varepsilon))$ and $M_{2}^{c}(\phi)=1-H_{c}(\phi)$

The level set function's regularity $\phi(\mathrm{x})$ is critical for stable evolution, and precise computation particularly close to the zero levels set position is expected. The effective fix is to apply re-initialization that is evolving to a steady-state defined by the partial differential equation in Eqn. 9

$$
\frac{\partial \phi}{\partial t}+\operatorname{sgn}\left(\phi_{0}\right)(|\nabla \phi|-1)=0, \phi(x, \tau=0)=\phi_{0}(x)
$$

where $\phi_{0}$ represents the level set function to be re-initialized. Nevertheless, $\phi_{0}$ does not remain steeper or smoother after multiple iterations. To skip high time-consumption during the re-initialization process, a level set regularization is formulated as in Eqn. 10.

$P(\phi)=\int_{\Omega} 1 / 2 \cdot(|\nabla \phi(x)|-1)^{2} d x$

Typically, during curve evolution, the penalty term induces the level set function $\phi$ near a signed distance function. Hence, the complete energy function is expressed as in Eqn. 11.

$$
\begin{gathered}
F\left(\phi, c_{1}, c_{2}\right)=\lambda_{1} \int_{\Omega}\left(\int K(x-y)\left(f(y)-c_{1}\right)^{2} d y\right) H_{s}(\phi(x)) d x+ \\
\lambda_{2} \int_{\Omega}\left(\int K(x-y)\left(f(y)-c_{2}\right)^{2} d y\right)\left(1-H_{s}(\phi(x))\right) d x \\
+\mu \cdot \int_{\Omega} \delta_{\varepsilon}(\phi(x))|\nabla \phi(x)| d x+v \int_{\Omega} 1 / 2 \cdot(|\nabla \phi(x)|-1)^{2} d x
\end{gathered}
$$

where $v$ is Lagrange multiplier coefficient and the positive constants are as shown in Eqn 12

$c_{1}(\phi)=\frac{\int_{0} f(x) H_{c}(\phi(x)) d x}{\int_{\Omega} H_{s}(\phi(x)) d x}, c_{2}(\phi)=\frac{\int_{\Omega} f(x)\left(1-H_{c}(\phi(x))\right) d x}{\int_{\Omega}\left(1-H_{s}(\phi(x))\right) d x}$

$\delta \varepsilon$ is the derivative of $H \varepsilon$ and is written as Eqn 13

$H_{\varepsilon}(z)=\frac{1}{2} \times\left(1+\frac{2}{\pi} \times \arctan \left(\frac{z}{\varepsilon}\right)\right), \delta_{s}=\frac{1}{\pi} \frac{\varepsilon}{x^{2}+\varepsilon^{2}}, \varepsilon>0$ 
On the Heaviside function's profile's smoothness, the parameter $\varepsilon$ generates an effect. Using a considerable value $\varepsilon$ allows the Heaviside function (regularized) to less steep close to the zero position, while expanding the range of capture and triggering less sensitive to blur boundary.

\section{Active Contours}

Given $U$ as a class of domains (regular bounded sets, open, $C_{2}$ ) of $R_{\mathrm{n}}\left(\mathrm{n}=2\right.$ or 3 ), and $\Omega_{i}$ an element of $U$ with boundary $q[\Omega i$, a segmentation problem (region-based) seeks at identifying a partition of $\Omega I$ in two regions $\{\Omega$ in,$\Omega$ out $\}$ that minimizes the criterion in Eqn 14.

$$
E\left(\Omega_{\text {in }}, \Omega_{\text {out }}, “\right)=J_{r}\left(\Omega_{\text {in }}\right)+J_{r}\left(\Omega_{\text {out }}\right)+\alpha J_{\text {reg }}(\text { “ })
$$

where $\operatorname{Jr}(\Omega i)$ illustrates the similarity of the region $\Omega i$ which can be categorized into sections $\mathrm{A}$ and $\mathrm{B}$ depending on the distribution, the expression $\Gamma$ between the two regions represents the common interface. The energy term Jreg is a regularization expression which is a balanced positive real parameter $\alpha$ selected as Jreg $=R \Gamma d a$, where $d a$ denotes an area element. In 2D, the term relates to minimizing the curve length while in $3 \mathrm{D}$ it minimizes the surface area. This term ensures a smooth curve or surface delineating the tumor. The shape gradient descent is performed by computing the evolution equation of an active contour by employing shape derivation tools. Computationally, the shape derivative deduced from the evolution equation to move the active contour is expressed in Eqn. 15.

$$
\frac{\partial “(p, \tau)}{\partial \tau}=v(x, \Omega) N(x)
$$

where $v(x, \Omega)$ is the active contour's velocity (using 2D or 3D) obtained from the shape derivatives with the velocity positioned along the unit inward normal $N$ of $\partial \Omega$. From an initial curve $\Gamma(\tau=0)=$ $\Gamma_{0}$, the active contour can evolve.

\section{Metrics}

As noted from the the review in Section 2, the most widely used metrics for performance evaluation are Dice Similarity Coefficient and the Jaccard Coefficient. In this study the performance of the standard Chan-Vese models is re-evaluated to assess is scalability on selected dataset of various forms of tumors. The dice similarity coefficient is primarily used as a quantitative measure of an algorithm's ability to automatically determine the segmentation accuracy. The Jaccard Coefficient on the other hand is used to measure the similarity of two sets. Their value ranges from 0 to 1 , where a value closer to one indicates acceptability. Mathematically, we have Eqn. 16 and Eqn. 17 to represent DSC and JC respectively.

Dice Similarity Coefficient $=\frac{2^{*} T P}{(T P+F P)+(T P+F N)}$

where FP, TP, TN, and FN represent false positive, true positive, true negative, and false negative, respectively. 


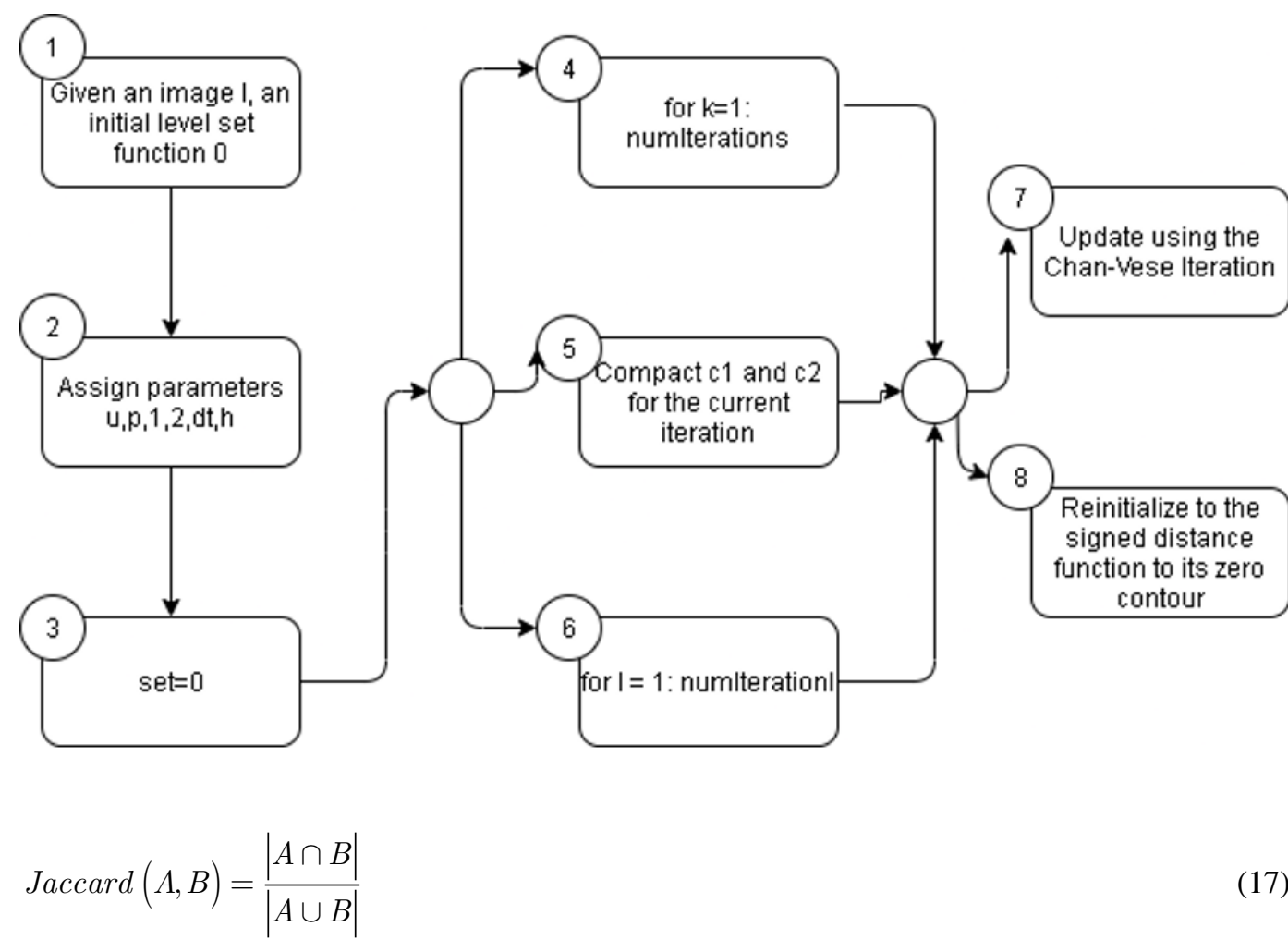

where $A$ and $B$ indicate the ground truth and segment result, respectively.

\section{The Chan-Vese Architecture}

The process flow diagram of the Chan-Vese architecture is shown in Figure 1. The first stage takes an initial image and establishes the contour. In the second stage, some variables are declared; the number of iterations of the model is set for the model to perform segmentation; the variables are initially declared with zero value. In the firth stage, the current iterations are displayed as segmentation occurs. In stage six, the iterations run according to the number set starting for one. In stage seven, the model records the current segmented results and in the last stage, after training, the model is ready to accept the next iteration value.

\section{RESULTS AND DISCUSSION}

This section discusses the performance of the Chan-Vese model in the detection of tumor from the brain, breast, and skin. The segmentation algorithm is trained and tested on an Intel ® Core (TM) i3-3220 CPU @ 3.30 GHz processor of three GigaHertz speed and eight GigaByte RAM (Random Access Memory) Dell Machine. Each analysis was performed at iteration 100, 150, 200, 250 and 300. Images with label "Y" are images with tumor present while "N" indicate images withour tumor.

\section{Observations with Brain Tumor Analysis}

Figure 2 illustrates the preprocessing phase. After performing segmentation on an image with a tumor, a Dice Similarity of 0.694993 , and Jaccard of 0.532558 was recorded. The elapsed time was 
Table 1. Performance Value on Brain Tumor Image

\begin{tabular}{|c|c|c|c|c|}
\hline Iteration & Dice Similarity & Jaccard & Elapsed Time & Image Name \\
\hline 100 & 0.694993 & 0.532558 & 7.389940 & Tumor Image 1 \\
\hline 150 & 0.694980 & 0.532543 & 11.043630 & Tumor Image 1 \\
\hline 200 & 0.694993 & 0.532558 & 12.817540 & Tumor Image 1 \\
\hline 100 & 0.598459 & 0.427001 & 9.831900 & Tumor Image 2 \\
\hline 150 & 0.598485 & 0.427027 & 13.925961 & Tumor Image 2 \\
\hline 200 & 0.598531 & 0.427074 & 16.637554 & Tumor Image 2 \\
\hline
\end{tabular}

7.389940 seconds at iteration 100, as shown in Figure 3. Figure 4 shows that of iteration 150 with Dice Similarity of 0.694980 , and Jaccard of 0.532543 at an elapsed time 11.043630. The iteration was stopped at 200 since there is no significant change as shown in Table 1.

After performing segmentation on an image without a tumor a Dice Similarity of 0.616987 , and a Jaccard of 0.446118 was recorded. The elapsed time was 16.556324 seconds at an iteration of 200, as shown in Figure 5.

\section{Breast Tumor Analysis}

With the breast tumor of the first image, after performing the segmentation, a Dice Similarity of 0.554107 , and Jaccard of 0.383228 was observed at an elapsed time of 9.577161 seconds with an iteration of 100, as shown in Figure 6. Repeating the process for iteration 150 and 200 with two test images we have Dice Similarity and Jaccard values recorded in Table 2.

After repeating the process again on a breast image without a tumor, a Dice Similarity of 0.518233 , and Jaccard of 0.349740 was recorded with an elapsed time of 11.232338 seconds at iteration 200 as shown in Figure 7.

Figure 2. Preprocessed Brain Tumor Image with Anisotropic Filter
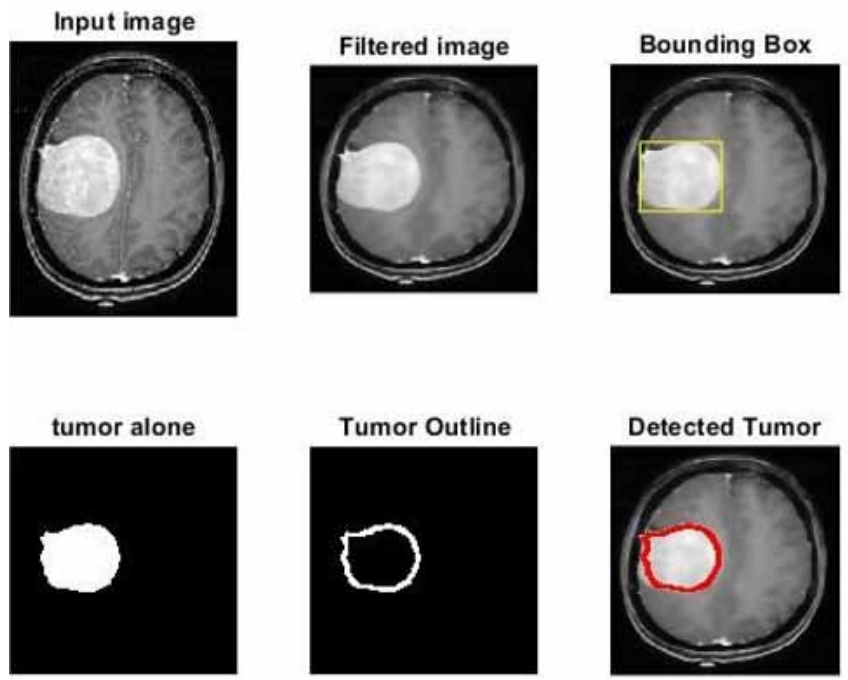


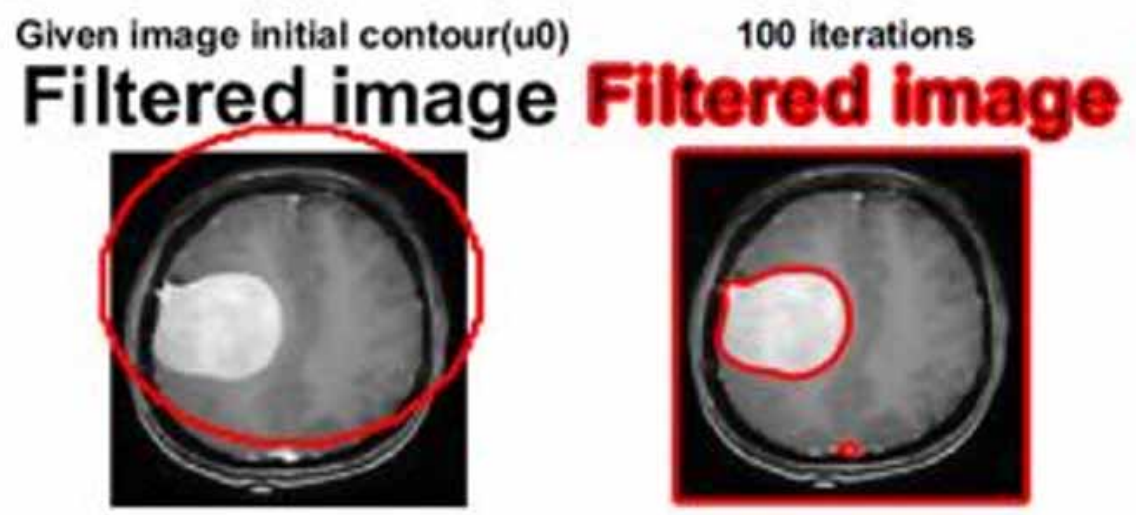

\section{Skin Tumor Analysis}

After performing segmentation on an image with a tumor, the model recorded a Dice Similarity of 0.618725 , and Jaccard of 0.447938 . The elapsed was 10.403951 seconds with an iteration of 100 , as shown in Figure 8. Repeating this process again at iteration 150 and 200, a Dice Similarity of 0.618719 and 0.618725 and a Jaccard of 0.447931 and 0.447938 were respectively obtained. Their computational time were noted to be 14.111079 and 16.480397 respectively at iteration 150 and 200. Figure 9 and Figure 10 represent the visual performance of the model as applied to a skinned image containing tumor. Applying the model on the second image with skin tumor, a Dice Similarity of 0.620293, and Jaccard of 0.449584 was recorded with an elapsed time of 9.597521 seconds at iteration 100 as shown

Figure 4. Chan-Vese Segmentation at Iteration 150 on Brain Tumor Image
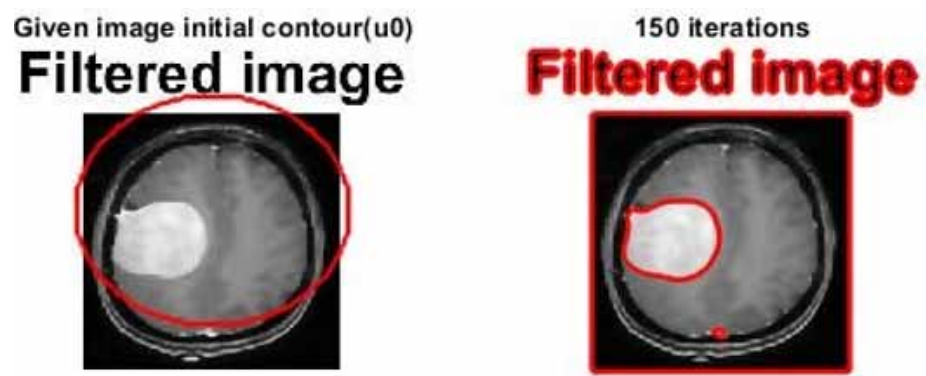

Figure 5. Chan-Vese Segmentation at Iteration 200 on Brain Tumor Image
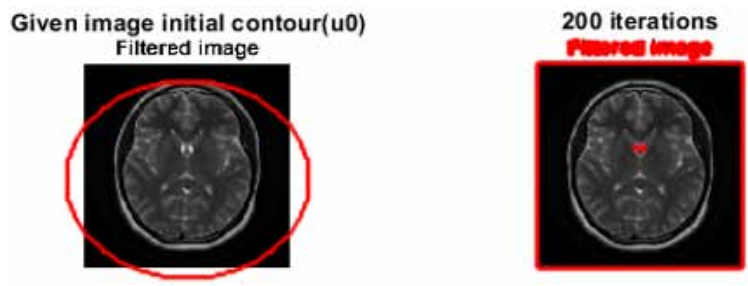
Table 2. Performance Value on Breast Tumor Image

\begin{tabular}{|c|c|c|c|c|}
\hline Iteration & Dice Similarity & Jaccard & Elapsed Time & Image Name \\
\hline 100 & 0.554107 & 0.383228 & 9.577161 & Tumor Image 1 \\
\hline 150 & 0.553814 & 0.382948 & 13.222634 & Tumor Image 1 \\
\hline 200 & 0.553696 & 0.382835 & 16.973739 & Tumor Image 1 \\
\hline 100 & 0.367115 & 0.537066 & 11.327466 & Tumor Image 2 \\
\hline 150 & 0.536902 & 0.366962 & 13.400257 & Tumor Image 2 \\
\hline 200 & 0.536852 & 0.366915 & 16.543606 & Tumor Image 2 \\
\hline
\end{tabular}

Figure 6. Chan-Vese Segmentation at Iteration 100 on Breast Tumor Image
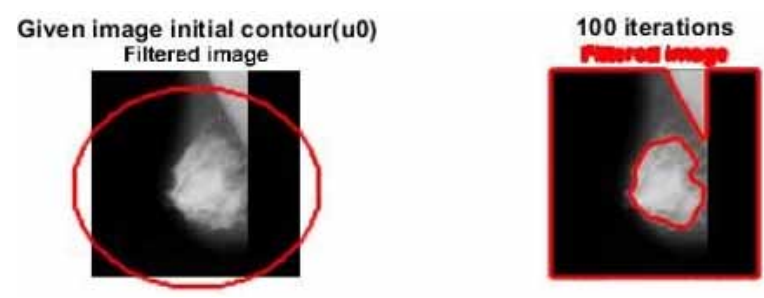

Figure 7. Chan-Vese Segmentation at Iteration 200 on Breast non-Tumor Image
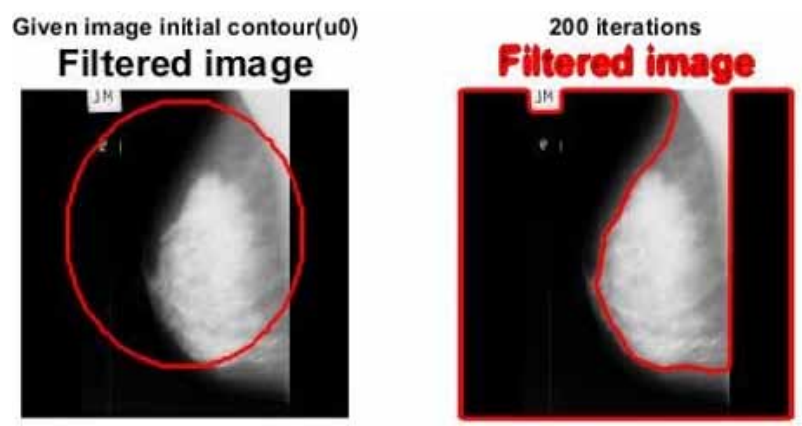

in Figure 11. At iteration 150 and 200, a Dice Similarity of (0.620350, 0.620420), and Jaccard of $(0.449643,0.449717)$ was recorded respectively at an elapsed time of $(13.496094,17.566681)$. The visual performance of the model at iteration 150 and 200 on the second image of the skin tumor is as shown in Figure 12 and Figure 13. Finally, this model recorded a Dice Similarity of 0.620680, and Jaccard of 0.449990 when applied on the skin image without a tumor cell. Computationally, it took 17.921264 seconds at iteration of 200 the generated the needed result.

\section{Discussion}

From the results reported for the brain, breast, and skin tumor, there is an indication that an increase in iterations do not necessarily affect performance, instead it only introduces time complexity at higher iterations. Using time as a performance indicator, it is observed that of all the tumors employed for this study, the Chan-Vese model performed relatively well on brain tumor segmentation, followed by breast 
Figure 8. Chan-Vese Segmentation at Iteration 100 on Skin Tumor Image
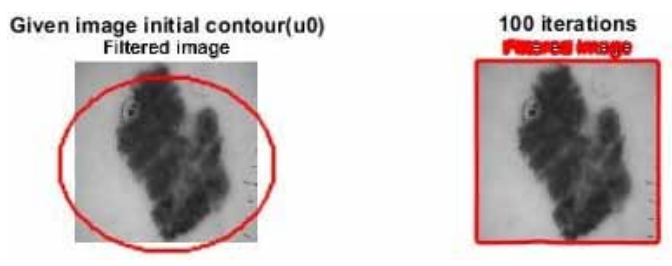

Figure 9. Chan-Vese Segmentation at Iteration 150 on Skin Tumor Image
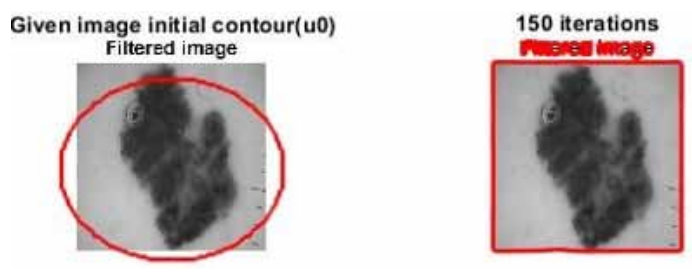

Figure 10. Chan-Vese Segmentation at Iteration 200 on Skin Tumor Image
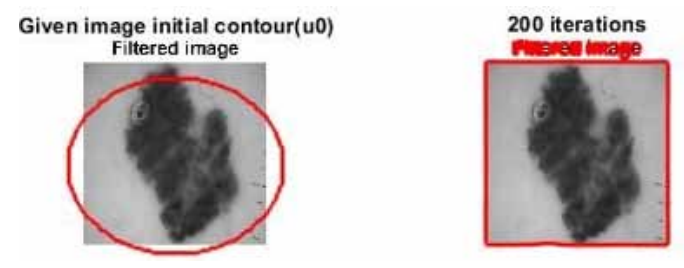

Figure 11. Chan-Vese Segmentation at Iteration 100 on Second Skin Tumor Image
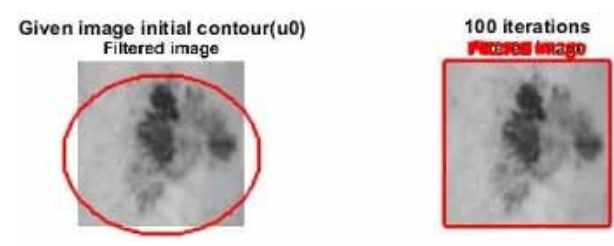

Figure 12. Chan-Vese Segmentation at Iteration 150 on Second Skin Tumor Image
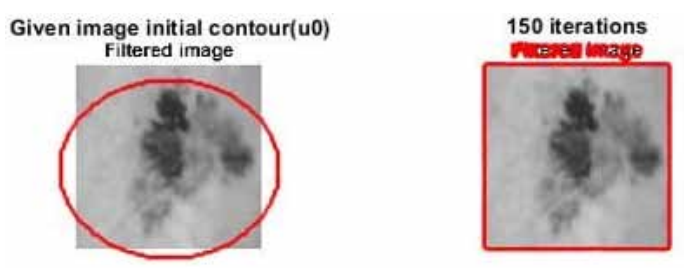
Figure 13. Chan-Vese Segmentation at Iteration 200 on Second Skin Tumor Image
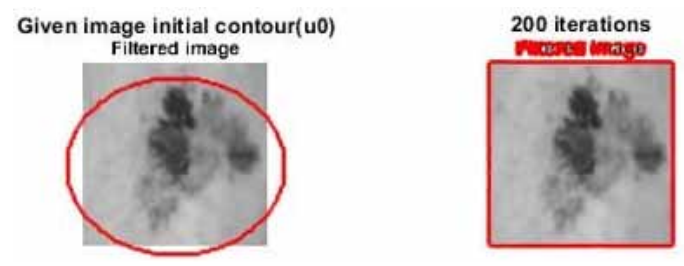

Table 3. Evaluation of the CV Technique

\begin{tabular}{|l|l|l|l|l|l|}
\hline \multicolumn{1}{|c|}{ Dataset } & \multicolumn{1}{|c|}{ Tumor } & \multicolumn{1}{|c|}{ Iteration } & Time Elapsed & \multicolumn{1}{c|}{$\begin{array}{c}\text { Dice Similarity } \\
\text { Coefficient }\end{array}$} & Jaccard \\
\hline BRAIN & WITH & 100 & 7.389940 & 0.6949903 & 0.532558 \\
\hline BREAST & WITH & 100 & 9.577161 & 0.554107 & 0.383228 \\
\hline SKIN & WITH & 200 & 17.566681 & 0.620420 & 0.449717 \\
\hline
\end{tabular}

tumor segementation and finally with skin tumor segmentation. Table 3 gives a summary evaluation of the Chan-Vese model indicating best performance on brain tumor in the light of disc similarity coefficient. The brain recorded an of disc similarity coefficient 0.6949903 , Jaccard 0.532558 , the time elapsed 7.389940 with an iteration of 100, The breast also recorded a DSC of 0.554107 , Jaccard 0.383228 , the time elapsed 9.577161 with an iteration of 100 . According to this study, a higher disc similarity coefficient does not signify a well-segmented image as the breast had a lower score than the skin yet performed better by visual inspection. The skin recorded a disc similarity coefficient of 0.620420 , Jaccard 0.449717, the time elapsed 17.566681 at an iteration of 200.

\section{CONCLUSION}

In conclusion, the study was able to segment tumor cells from MR/CT images using the MIAS, Navoneel, and ISIC dataset to investigate the performance scalability of the well known ChanVese model. The datasets involved images consisting of tumors of the brain, breast, and skin. From the analysis of the various datasets, the Chan-Vese could be rated high only when applied to the segmentation of brain tumors other than the other forms of tumors like the breast and skin. This conclude that though the Chan-Vese model is known to be very good for tumor detection it does not scale across all tumor type. Moving forward other models known to work well should be further investigated on their scalability. Furthermore, hybrid models can also be considered for investigation 
an assess their scalability. It is important to obtain models that scales well with some predefined parameter to better build a unified diagnostic system.

\section{REFERENCES}

Abbasi, S., \& Tajeripour, F. (2017). Detection of brain tumor in 3D MRI images using local binary patterns and histogram orientation gradient. Neurocomputing, 219, 526-535. doi:10.1016/j.neucom.2016.09.051

Badshah, N., Rabbani, H., \& Atta, H. (2020). On local active contour model for automatic detection of tumor in MRI and mammogram images. Biomedical Signal Processing and Control, 60, 101993. doi:10.1016/j. bspc. 2020.101993

Bal, A., Banerjee, M., Sharma, P., \& Maitra, M. (2018). Brain tumor segmentation on MR image using K-means and fuzzy-possibilistic clustering. 2018 2nd International Conference on Electronics, Materials Engineering and Nano-Technology, IEMENTech 2018, 1-8. doi:10.1109/IEMENTECH.2018.8465390

Chahal, P. K., Pandey, S., \& Goel, S. (2020). A survey on brain tumor detection techniques for MR images. Multimedia Tools and Applications, 79(29-30), 21771-21814. doi:10.1007/s11042-020-08898-3

Gao, Y., Shao, Y., Lian, J., Wang, A. Z., Chen, R. C., \& Shen, D. (2016). Accurate Segmentation of CT Male Pelvic Organs via Regression-Based Deformable Models and Multi-Task Random Forests. IEEE Transactions on Medical Imaging, 35(6), 1532-1543. doi:10.1109/TMI.2016.2519264 PMID:26800531

Gosse, K., Jehan-Besson, S., Lecellier, F., \& Ruan, S. (2017). Comparison of 2D and 3D region-based deformable models and random walker methods for PET segmentation. 2016 6th International Conference on Image Processing Theory, Tools and Applications, IPTA 2016. doi:10.1109/IPTA.2016.7820959

Khalil, H. A., Darwish, S., Ibrahim, Y. M., \& Hassan, O. F. (2020). 3D-MRI brain tumor detection model using modified version of level set segmentation based on dragonfly algorithm. Symmetry, 12(8), 1256. Advance online publication. doi:10.3390/sym12081256

Kharote, P. R., Sankhe, M. S., \& Patkar, D. (2019a). Automatic Segmentation of Prostate from Multiparametric MR Images Using Hidden Features and Deformable Model. IEEE Region 10 Annual International Conference, Proceedings/TENCON, 338-343. doi:10.1109/TENCON.2019.8929686

Kharote, P. R., Sankhe, M. S., \& Patkar, D. (2019b). Prostate segmentation and tumor detection from MR images using latent features. 2019 IEEE 16th India Council International Conference, INDICON 2019 - Symposium Proceedings, 0-3. doi:10.1109/INDICON47234.2019.9030316

Kozegar, E., Soryani, M., Behnam, H., Salamati, M., \& Tan, T. (2018). Mass Segmentation in Automated 3-D Breast Ultrasound Using Adaptive Region Growing and Supervised Edge-Based Deformable Model. IEEE Transactions on Medical Imaging, 37(4), 918-928. doi:10.1109/TMI.2017.2787685 PMID:29610071

Kumar, S., Jayadevappa, D., \& Shetty, M. V. (2018). A novel approach for segmentation and classification of brain MR images using cluster deformable based fusion approach. Periodicals of Engineering and Natural Sciences, 6(2), 237-242. doi:10.21533/pen.v6i2.271

Kumar, S., \& Mankame, D. P. (2020). Optimization driven Deep Convolution Neural Network for brain tumor classification. Biocybernetics and Biomedical Engineering, 40(3), 1190-1204. doi:10.1016/j.bbe.2020.05.009

Lakshmi Priya, B., Saraswathi, D., \& Punitha Lakshmi, R. (2019). Liver segmentation using weighted contrast based chan-vese method. 2019 IEEE International Conference on System, Computation, Automation and Networking, ICSCAN 2019, 1-5. doi:10.1109/ICSCAN.2019.8878810

Lee, W. L., Chang, K., \& Hsieh, K. S. (2016). Unsupervised segmentation of lung fields in chest radiographs using multiresolution fractal feature vector and deformable models. Medical \& Biological Engineering \& Computing, 54(9), 1409-1422. doi:10.1007/s11517-015-1412-6 PMID:26530048

Mohamed Sathik, M., \& Synthiya Judith Gnanaselvi, E. (2019). An efficient brain tumor classification based on sobs method for MRI brain images. International Journal of Engineering and Advanced Technology, 9(1), 826-833. doi:10.35940/ijeat.A9379.109119 
Namías, R., D’Amato, J. P., del Fresno, M., Vénere, M., Pirró, N., \& Bellemare, M. E. (2016). Multi-object segmentation framework using deformable models for medical imaging analysis. Medical \& Biological Engineering \& Computing, 54(8), 1181-1192. doi:10.1007/s11517-015-1387-3 PMID:26392182

Pérez-Ramírez, Ú., Arana, E., \& Moratal, D. (2016). Brain metastases detection on MR by means of threedimensional tumor-appearance template matching. Journal of Magnetic Resonance Imaging, 44(3), 642-652. doi:10.1002/jmri.25207 PMID:26934581

Rajendran, A., \& Dhanasekaran, R. (2012). Fuzzy clustering and deformable model for tumor segmentation on MRI brain image: A combined approach. Procedia Engineering, 30, 327-333. doi:10.1016/j.proeng.2012.01.868

Sehgal, A., Goel, S., Mangipudi, P., Mehra, A., \& Tyagi, D. (2016). Automatic brain tumor segmentation and extraction in MR images. Conference on Advances in Signal Processing, CASP 2016, 104-107. doi:10.1109/ CASP.2016.7746146

Sharif, M., Amin, J., Raza, M., Anjum, M. A., Afzal, H., \& Shad, S. A. (2020). Brain tumor detection based on extreme learning. Neural Computing \& Applications, 8(20), 15975-15987. Advance online publication. doi:10.1007/s00521-019-04679-8

Shil, S. K., Polly, F. P., Hossain, M. A., Ifthekhar, M. S., Uddin, M. N., \& Jang, Y. M. (2017). An improved brain tumor detection and classification mechanism. International Conference on Information and Communication Technology Convergence: ICT Convergence Technologies Leading the Fourth Industrial Revolution, ICTC 2017, 54-57. doi:10.1109/ICTC.2017.8190941

Shivhare, S. N., Kumar, N., \& Singh, N. (2019). A hybrid of active contour model and convex hull for automated brain tumor segmentation in multimodal MRI. Multimedia Tools and Applications, 78(24), 34207-34229. doi:10.1007/s11042-019-08048-4

Shrivastava, N., \& Bharti, J. (2020). Breast tumor detection and classification based on density. Multimedia Tools and Applications, 79(35-36), 26467-26487. Advance online publication. doi:10.1007/s11042-020-09220-x

Sultana, S., Blatt, J. E., Gilles, B., Rashid, T., \& Audette, M. A. (2017). MRI-Based Medial Axis Extraction and Boundary Segmentation of Cranial Nerves Through Discrete Deformable 3D Contour and Surface Models. IEEE Transactions on Medical Imaging, 36(8), 1711-1721. doi:10.1109/TMI.2017.2693182 PMID:28422682

Vorontsov, E., Tang, A., Roy, D., Pal, C. J., \& Kadoury, S. (2017). Metastatic liver tumour segmentation with a neural network-guided 3D deformable model. Medical \& Biological Engineering \& Computing, 55(1), $127-139$. doi:10.1007/s11517-016-1495-8 PMID:27106756

Wang, Z., Wang, K., Yang, F., Pan, S., \& Han, Y. (2018). Image segmentation of overlapping leaves based on Chan-Vese model and Sobel operator. Information Processing in Agriculture, 5(1), 1-10. doi:10.1016/j. inpa.2017.09.005

Zawish, M., Siyal, A. A., Ahmed, K., Khalil, A., \& Memon, S. (2019). Brain tumor segmentation in MRI images using Chan-vese technique in MATLAB. 2018 International Conference on Computing, Electronic and Electrical Engineering, ICE Cube 2018, 1-6. doi:10.1109/ICECUBE.2018.8610987 
Justice Kwame Appati is a lecturer in the School of Physical and Mathematical Science (SPMS) and the Department of Computer Science. He began his teaching career at Kwame Nkrumah University of Science and Technology in Kumasi as a graduate assistant and then later moved to the University of Ghana in 2017 as a lecturer. Justice earned a PhD, in Applied Mathematics from Kwame Nkrumah University Science and Technology in 2016. He also graduated in 2010 and 2013 with a BSc. Mathematics and MPhil Applied Mathematics from the same institution. His current research includes data science, mathematical intelligence, image processing and scientific computing, He has singly and jointly supervised undergraduate and postgraduate students from Kwame Nkrumah University of Science and Technology (KNUST), National Institute of Mathematical Sciences (NIMS), African Institute of Mathematical Sciences (AIMS) and the University of Ghana Currently, Justice handles course like Design and Analysis of Algorithm, Artificial Intelligence, Formal Methods and Computer Vision. He looks forward to working with everyone interested in his field of study more especially, Intelligence and Data Science.

Franklin Iron Badzi is a teacher at the Yilo Krobo Senior High School department of Information and Communication Technology. Mr Badzi taught computer related course in Afife Secondary Technical and Mount Mary College of Education Somanya. His current research interest include Parallel Computing, Ubiquitous Computing, Neural Networks, Data Mining and Artificial intelligence. He received the regional Best Teacher's award in Science and Mathematics Junior Category in the Volta Region of Ghana. Born in Aflao V/R Ghana, Mr. Badzi earned his B.Sc in Information Technology from University of Education Winneba Kumasi campus.

Michael Agbo Tettey Soli is a critical thinker and solutions engineer with over a decade of experience in software engineering and algorithm design. He is currently a Ph.D. student with the Department of Computer Science of the University of Ghana.

Stephane Nwolley Jnr. holds a PhD in ICT Management (Big Data), with high-level expertise in synthesizing large amounts of information into insights for strategy, innovation, and business development. He was highly instrumental in creating an automated machine learning tool (snwolley) designed to make data analytics easy and affordable. He has had training in project management, Master of Business Administration in information systems from SMU, and Bachelor of Applied Computer Science from Royal Melbourne Institute of Technology, Australia. His key qualities are in Big Data, Data Science, Cloud Computing, Business intelligence and ERP, UNIXI LINUX Programming, Web Application Design and Development, Project Management and Risk Analysis, Human Computer Interaction, DB Management skills and Operating System Administration. Dr. Stephane Nwolley Jnr. has more than 14 years of practical experience in the ICT industry and previously worked with MTN and Huawei as an OSS engineer. His experience and hands-on knowledge of the industry enables him to proffer solutions that are both practical and innovative. Formerly, the CEO of Nalo Solutions Limited, he was the product lead for projects with clients including Ecobank, GIPC, UBA, amongst others. He currently serves as an adjunct lecturer in the Computer Science department of Ashesi University. With the distinction of being an academic and an entrepreneur, he is noted for his skill in breaking down complex conceptual frameworks into simple, practical and useful terms. He has contributed to the Big data and Al conversations through publications. Dr. Stephane Nwolley Jnr. is Founder and CEO of Npontu Technologies, an IT company with three integrated product offerings, Big Data and Machine Learning, Software development and Value-Added Services. His company currently provides service for organizations like GCB Bank, Stanbic Bank, Standard Chattered Bank, Cal Bank, Access Bank, Enterprise Insurance, Vanguard Assurance, Allianz Insurance, Goil, Total Petroleum, National Identification Authority, Office of the President, USAID, PWC just to name a few.

Ismail Wafaa Denwar is an IT Officer at Ghana Electrometer Limited, an electricity manufacturing company supplying the Electricity Company of Ghana (ECG) with electricity meters; he has been working there since 2017. He obtained a Bachelor of Science in Information Technology degree at Valley View University, Accra, Ghana, from 2013 to 2017. Mr. Ismail proceeded to the University of Ghana, which led to an award of a Master of Science in Computer Science degree from 2019 to 2020, running concurrently with his job at Electrometer. He is currently assisting in the publication of research papers in other journals. His research interest is in areas of Neural Networks (Text Generation, Text Classification, Time-series forecast), Machine Learning (Classification problems such as intrusion detection based on network traffic among others), Databases, and Image Processing (particularly tumor detections). Mr. Ismail has a strong passion for research and is looking forward to doing a Ph.D. in the future; he has a passion for finding answers to solving complex problems. 